\title{
Umkämpfte Orte
}

Rezension zu Anne Huffschmid (2015): Risse im Raum: Erinnerung, Gewalt und städtisches Leben in Lateinamerika. Wiesbaden: Springer VS.

In seiner Theorie des Filmes erzählt Siegfried Kracauer den Mythos der Enthauptung der Medusa durch Perseus nach, um das Problem der Sichtbarkeit und Darstellbarkeit von Terror zu veranschaulichen (2005 [1964]: 467-468). Nach Kracauer verkörpert der Anblick der Medusa reinen Terror und dessen versteinernde Kraft. Dem griechischen Mythos zufolge kann Perseus die Medusa nur enthaupten, weil er sie nicht direkt, sondern allein ihr Spiegelbild auf dem Schild, das ihm die Göttin Athene leiht, anschaut. Dies suggeriere - so Kracauer -, dass der Horror nur durch dessen Repräsentation besiegt werden könne. Darüber hinaus betont er, dass Athene den Kopf der Medusa auf ihrem Schild befestige, um an den Schrecken und an dessen Besiegbarkeit zu erinnern.

Entlang der Fragen nach der Darstellung und der Erinnerung an den argentinischen und mexikanischen Staatsterror der 1970er-Jahre entwickelt Anne Huffschmid Risse im Raum: Erinnerung, Gewalt und städtisches Leben in Lateinamerika. Um den Fokus ihrer ethnologischen Untersuchung zu schärfen, konzentriert sie sich auf städtische Räume, die sie als veränderliche Produkte sozialer und politischer Auseinandersetzungen begreift. Programmatisch sucht sie nach denjenigen Rissen, die die Verfassung des Stadtraumes destabilisieren und seine soziale Bedingtheit offen legen. Ihrer Meinung nach generiert die Erinnerung an Gewalt Brüche, die die palimpsestartige Natur des Raumes offenbaren. In den Rissen verschränkten sich unterschiedliche Zeiten: die gegenwärtige Praxis des Erinnerns, die erinnerte Vergangenheit und die utopische Zukunft des ,nunca más', des ,Nie-Wieder‘ Darüber hinaus zeigen - der Autorin nach - solche Brüche nicht nur verschränkteZeitschichten, sondern auch, dass durch konkurrierende Praktiken und Interpretationen des Raumes um Deutungshoheit gekämpft wird. Die erinnernde Praxis mache „[...] im Strom des Urbanen für einen Moment lang das Ungeheuerliche spürbar [...]“, störe dadurch die sozialen Routinen und mache die Bedingtheit des Raumes sichtbar (Huffschmid 2015: 462).

Im ersten - theoretischen - Teil von Risse im Raum verbindet die Autorin diverse Diskussionen und Positionen. Sie präsentiert nicht nur Debatten aus unterschiedlichen Disziplinen, sondern bewegt sich zudem souverän 
zwischen verschiedenen sprachlichen und regionalen Kontexten. Dabei führt sie die Arbeit der Raumtheoretiker Michel de Certeau und Henri Lefebvre sowohl mit erinnerungskulturellen Ansätzen von Aleida Assmann zusammen als auch mit den repräsentationstheoretischen Fragestellungen von Roland Barthes und Georges Didi-Hubermann. Darüber hinaus bringt Huffschmid spanischsprachige Werke wie die von Manuel Delgado und Armando Silva mit deutschsprachigen Beiträgen zur Erinnerungskultur in Verbindung. Ein Beispiel dafür liefert der in der Studie zentrale Begriff des imaginario. Er wurde unter anderem von Armando Silva (2006 [1992]) geprägt, um kollektive Horizonte, in denen sich das Immaterielle mit dem Materiellen der städtischen Erfahrung verbinde, besser beschreiben zu können. Die Idee des imaginario impliziert einerseits eine verstärkte Betrachtung der Materialität von Erinnerung und damit die Räumlichkeit derselben, andererseits schließt der Begriff in das materielle Feld des Raumes auch rein diskursive Elemente ein. Somit wird eine verschränkte Betrachtung von Materialität und Diskursen möglich.

Der theoretische Beitrag der Autorin geht über diesen ersten Teil hinaus, indem sie sich im letzten Kapitel vor den Schlussbemerkungen erneut mit theoretischen Fragen beschäftigt. In diesem zusätzlichen Kapitel, das auf den empirischen Teil folgt und wie ein Anhang erscheint, setzt sie sich mit dem ,Wissen der Bilder' auseinander. Hier thematisiert sie sowohl ihre Verwendung der Bilder als Forschungsquelle und -praxis, als auch die „Medusa-Frage‘ nach der bildlichen Darstellung des Schrecklichen. Bezüglich dieser zweiten Frage nimmt Huffschmid Stellung „gegen eine verbreitete Rhetorik von der Unvorstellbarkeit des Terrors, die zur Bildlosigkeit verdammt, da vermeintlich jede Visualisierung den Vorgang des Erinnerns wie auch das Erinnerte selbst trivialisiere“ (2015: 376). Diese Position arbeitet sie vor allem anhand der Debatten zur Darstellbarkeit der Shoah (DidiHubermann 2007) und des lateinamerikanischen Staatsterrorismus (Garcia/ Longoni 2012) heraus. Sie unterstreicht die Parallelen dieser Diskussionen und leistet damit einen wertvollen Beitrag zur Einbindung spanischsprachiger in deutschsprachige Debatten. Methodologischen Fragestellungen zum ,Wissen der Bilder in der Ethnographie begegnet Huffschmid durch eine vielfältige Einbettung ihrer eigenen Position in die bereits existierende Literatur. So zeigten Bilder „[...] unser eigenes Sehen und ihm innewohnende, partiality', in dem sie uns vor Augen führen, welche Ausschnitte des Feldes wir aus welcher Einstellung im Blick haben und welche gerade nicht" (2015: 405). Aus diesem Grund ermögliche das Produzieren und das Lesen von Bildern die nötige Distanz, die die subjektive Position des Schauenden sichtbar mache. Diese subjektive Position konzipiert Huffschmid gleichzeitig als partiality und als Unterworfen-Sein des Subjektes unter Praktiken und Diskurse. Durch die technische Schaffung einer bildlichen Materialität, die sowohl eigen als auch fremd sei, stelle die Fotografie die partielle und unterworfene Natur des subjektiven Blickes bloß und ermögliche so, den Blick für das ,Andere' zu öffnen. Genau wie im ersten theoretischen Teil geht die Autorin auch hier geschickt mit heterogenen Debatten um. Trotzdem bleibt die Entscheidung, diese Themen - die auch stark ihre Forschungsmethoden beeinflussen - nach der Empirie zu präsentieren, etwas unverständlich. Die Lesenden bekommen dadurch den Eindruck, einen nachträglich verfassten 
Anhang zu lesen, der von den zentralen Fragestellungen losgelöst ist und sich wie einen Streifzug durch poststrukturalistische Theorien anfühlt.

Der empirische Teil von Risse im Raum ist das Ergebnis einer vielfältigen ethnographischen Arbeit, die sich über fast ein Jahrzehnt erstreckte (2005 bis 2013) und für die die Autorin Buenos Aires und Mexiko-Stadt in regelmäßigen Abständen besuchte. Die Ergebnisse dieser langjährigen Forschung sind vor allem Feldnotizen, Interviews, Fotos, Archivdokumente und Artikel. Da Huffschmid ihren Blick insbesondere auf die Entstehung von kontroversen Erinnerungsräumen lenkt, beziehen sich die Feldnotizen hauptsächlich auf ihre Beobachtungen der räumlichen Materialitäten und Praktiken, die mit den ausgesuchten Orten verbunden sind. Die Interviews hingegen umfassen ein breiteres Feld, in dem individuelle Erinnerungen sowie Strategien der Erinnerungspolitik behandelt werden. Die zahlreichen Interviewpartner_innen in Argentinien und Mexiko sind vor allem Betroffene oder Aktivist_innen, die sich mit der Erinnerungspolitik zum Staatsterrorismus beschäftigen. Das hochinteressante Material wird durch Fotos und die Rekonstruktion politischer Debatten ergänzt und konterkariert. Teilweise ordnet Huffschmid etwas verkürzt - aber deswegen nicht uninteressant - sowohl den argentinischen und mexikanischen Staatsterror als auch unterschiedliche erinnerungspolitische Positionen den nationalen Meistererzählungen der zwei Nationen zu.

In den zwei Städten identifiziert die Autorin hauptsächlich vier verschiedene Typologien von Erinnerungsorten: Geheimgefängnisse und Gedenkstätten, öffentliche Plätze, Gerichte als ,Räume des Rechts‘ sowie Orte der Kunst. Exemplarisch werden im Folgenden einige Beispiele kurz erläutert, die zugleich die Hauptargumente veranschaulichen. Im Fall von Buenos Aires sind die analysierten Geheimgefängnisse und Gedenkstätten das Gelände der ESMA (Escuela de Mecánica de la Armada), der Club Atlético und die Garage Olimpo. Insbesondere anhand des ESMA-Areals gelingt es der Autorin die konflikthafte Natur von Erinnerungsräumen und die Auseinandersetzung um die Repräsentation des Terrors zu zeigen. Die ESMA, die ursprünglich als Akademie der argentinischen Marine fungierte, wurde während der Militärdiktatur teilweise als Haft- und Folterlager benutzt: „Das Gelände, das im März 2004 offiziell zum ,Erinnerungsraum` umgewidmet wurde, gilt heute als Emblem einer erfolgreich institutionalisierten und stadträumlich etablierten Erinnerungspolitik“ (Huffschmid 2015: 123). Die Autorin erläutert weiterhin, wie unterschiedliche Akteure - darunter die Stadt- und Bundesregierung sowie zahlreiche Aktivistengruppen und -vereine - das große Gelände mit teilweise antagonistischen, aber auch komplementären Deutungen und Praktiken besetzen. Am Beispiel der ESMA zeige sich so die Zerrissenheit und das Umkämpft-Sein von Erinnerungsräumen, die als instabiles Palimpsest immer wieder überschrieben und umgedeutet werden.

Das mexikanische Pendant ist in diesem Fall etwas weniger aussagekräftig: der Campo Militar Nr. 1 fungiert heute immer noch als Militärstützpunkt und wurde seit den späten 1960er-Jahren bis in die 1970er-Jahre teilweise als Geheimgefängnis benutzt. Eine der Interviewpartnerinnen definiert den Ort als „kein[en] Erinnerungsort, [sondern] eher ein[en] ,Ort der Amnesie“, da es hier offensichtlich keinen politischen Willen zur Erinnerung [gibt]“ (Huffschmid 2015: 171). Wie Huffschmid herausstellt, versucht eine kleine 
Gruppe von Aktivist_innen den Ort als Erinnerungsraum zu deuten, ohne dass es ihnen gelänge die Deutungshoheit zu erlangen.

Allgemein ist der Vergleich zwischen den zwei Städten zwar - wie von der Autorin selbst deklariert - problematisch, aber trotzdem nicht uninteressant. Buenos Aires und Mexiko-Stadt stellt sie als „diametrale Pole lateinamerikanischer Geschichtskultur“ (2015: 16) dar. Während in Buenos Aires die Erinnerung an den Staatsterror in vielen Räumen wachgehalten und auch von offiziellen politischen Institutionen getragen werde, habe die Erinnerung an Gewalt in Mexiko-Stadt keinen stabilen Stand. Huffschmids Ausgangpunkt ist, dass „vergleichbare Gewalterfahrungen - die staatlich organisierte Repression gegen die politische und bewaffnete Opposition in den 1970er-Jahren - [sich] höchst unterschiedlich im öffentlichen und städtischen Raum materialisieren“ (ebd.). Die Vergleichbarkeit der mexikanischen und argentinischen Gewalterfahrung in den 1970er-Jahren vorauszusetzen, ist dennoch etwas problematisch. Auch wenn sich die gewalttätigen staatlichen Praktiken des Verschwinden-Lassens in Argentinien und Mexiko ähnelten, unterschieden sie sich extrem bezüglich ihres Ausmaßes, ihres politischen Rahmens, ihrer öffentlichen Resonanz und deshalb auch in ihrem Einfluss auf das öffentliche Leben und den städtischen Raum. Während man in - vom Militär regierten - Argentinien von einer Zahl zwischen 12.00o und 22.00o Verschwundenen ausgeht, schätzt man die Zahl der desaparecidos in - von gewählten Zivilist_innen regierten - Mexiko auf ungefähr tausend. Angesichts dieser Tatsachen scheinen die Ergebnisse des Vergleiches in Risse im Raum bereits durch die Auswahl der erforschten Fälle etwas vorprogrammiert: Buenos Aires wird als Hochburg des Ringens um Erinnerung dargestellt, während Mexiko-Stadt auf die Leser_innen wie eine Bühne für den einsamen Kampf einiger weniger Betroffener und Aktivist_innen wirkt. Trotz dieser Probleme, die aber auch von der Autorin thematisiert werden, machen ihr angenehmer Schreibstil und ihr analytisches Feingefühl sowohl die argentinischen als auch die mexikanischen Fälle interessant.

Weitere Fallstudien sind die,öffentlichen Plätze‘ Plaza de Mayo in Buenos Aires und Plaza Tlatelolco in Mexiko-Stadt. Letzterer wird auch ,Platz der drei Kulturen' genannt, weil auf ihm die Ruinen einer präkolumbianischen Pyramide, einer Kirche im spanisch-kolonialen Stil und einer modernen Wohnsiedlung der 1960er-Jahre zu finden sind. Diese drei Bauten sollen die indigene, die spanische und die moderne (mexikanische) Kultur symbolisieren, die die Hauptbestandteile der nationalen Meistererzählung ausmachen. Auf dem Platz ereignete sich am 2. Oktober 1968 ein blutiges Massaker, bei dem mehrere Hundert unbewaffnete Student_innen von regierungsnahen Gruppen ermordet wurden. 1985 stürzte in seiner unmittelbaren Nähe ein ganzes Hochhaus aufgrund eines Erdbebens ein. Der Platz - der jährlich Ausgangspunkt eines Erinnerungszugs für die Opfer des Studentenmassakers ist - zeige nach Huffschmid die „konfliktive Gleichzeitigkeit, [in der] verschiedene Verbindungen zwischen Vergangenheit und Gegenwart zum Vorschein [kommen]“ (2015: 197). In die Plaza Tlatelolco hätten sich „neben dem Massaker die Erfahrungen zweier weiterer - höchst unterschiedlicher Katastrophen eingelagert: die Niederlage des Aztekenkriegers Cuauhtémoc, der hier im Jahr 1521 die letzte Schlacht gegen die spanischen Invasoren 
verlor, sowie das verheerende Erdbeben von 1985“ (ebd.: 198). Während Huffschmid das Funktionieren der räumlichen Erinnerungspraxis an das Studentenmassaker klar und ausführlich zeigt, wird die Erinnerung an Cuauhtémoc etwas unkommentiert vorausgesetzt. Das Verhältnis zwischen der Erinnerung an koloniale Gewalt und an Gewalt im 20. Jahrhundert und deren ,Einlagerung' wird nicht weiter erklärt.

Auch die Plaza de Mayo in Buenos Aires ist ein geschichtsträchtiger Ort. Der Platz war und ist der zentrale Schauplatz der argentinischen Hauptstadt und wurde in unterschiedlichen historischen Phasen - von der Stadtgründung bis heute - von vielfältigen teilweise konkurrierenden Funktionen, Praktiken und Deutungen geprägt. Die Plaza de Mayo sei „seit mindestens zwei Jahrhunderten der politische Platz der Stadt“ (Huffschmid 2015: 224), wo heute die Casa Rosada (Sitz des Präsidenten), die nationale Notenbank, die Kathedrale und der Hauptsitz der Stadtverwaltung angesiedelt sind. Die Autorin geht in ihrer Erzählung vor allem auf den Prozess ein, der es den ,Müttern der Plaza de Mayo، erlaubt habe, diesem Raum einen neuen Sinn zu verleihen und ihn als politischen Schauplatz für sich zu erobern. Im Jahr 1977 - noch während der Militärdiktatur - traf sich eine Gruppe von Müttern der desaparecidos und veranstaltete einen Rundgang um den Obelisken der Mairevolution, der den Unabhängigkeitskampf gegen die spanische Kolonialmacht symbolisiert. Die Frauen benutzten den national und bürgerlich kodierten Raum und die in der Militärdiktatur essenzialisierte Figur der Mutter strategisch, um auf die Gewalt in der Diktatur aufmerksam zu machen und um Räume für Widerstand zu öffnen. So zeigt sich im Fall der Plaza de Mayo erneut, wie ein - von Erinnerung und Geschichte besetzter - Raum durch politische Praktiken umgedeutet werden kann. Der Fall der Plaza de Mayo verdeutlicht im argentinischen Kontext auch die komplexe Schichtung von Räumen, in denen sich nicht nur zeitliche, sondern auch politische - teilweise konkurrierende - Deutungen und Praktiken verschränken.

Jenseits dieser Beispiele präsentiert Huffschmid weitere Fälle, deren Darlegung sich über zweihundert Seiten erstreckt. Auch wenn sie im Einzelnen spannende Fragen aufwerfen und interessant präsentiert werden, leidet der Lesefluss unter der übermäßigen Materialfülle, wodurch auch die Prägnanz der Fragestellung teilweise in der Menge des Erzählten verschwindet.

Trotz einiger struktureller Probleme ist dieses gutrecherchierte Buch eine lehrreiche Lektüre für Leser_innen, die sich mit der Erinnerungskultur, der lateinamerikanischen Geschichts- und Erinnerungspolitik und der Stadtforschung in Bezug auf Erinnerung beschäftigen. Huffschmid leistet darin eine theoretische Verbindung zwischen Debatten und Theorien aus unterschiedlichen Regionen und Disziplinen. Die Klarheit, mit der sie ihren Leser_innen diese vielfältigen Diskussionen erklärt, macht das Buch auch über eine reine Fachleserschaft hinaus zugänglich und lesenswert. Risse im Raum: Erinnerung, Gewalt und städtisches Leben in Lateinamerika ist somit eine neue interessante Referenz im deutschsprachigen Raum, die nicht nur zu einem besseren Verständnis von Buenos Aires und Mexiko-Stadt, sondern auch zur konzeptuellen Verschränkung von Raum und Zeitlichkeit in Städten beiträgt. 


\section{Autor_innen}

Antonio Carbone ist Historiker. Er forscht zur Globalgeschichte und zur lateinamerikanischen und europäischen Urbanisierungsgeschichte des 19. und 20. Jahrhunderts.

antonio.carbone@fu-berlin.de

\section{Literatur}

Assmann, Aleida (1999): Erinnerungsräume: Formen und Wandlungen des kulturellen Gedächtnisses. München: C.H. Beck.

Assmann, Aleida / Conrad, Sebastian (Hg.) (2010): Memory in a Global Age: Dirscourses, Practices and Trajectories. Basingstoke: Palgrave Macmillan.

Barthes, Roland (2012 [1980]): Die helle Kammer. Bemerkungen zur Photographie. Frankfurt am Main: Suhrkamp.

Brodsky, Marcelo (Hg.) (2005): Memoria en construcción: El debate sobre la ESMA. Buenos Aires: La Marca Editorial.

Delgado, Manuel (1999): El animal público: hacia una antropología de los espacios urbanos. Barcelona: Editorial Anagrama.

Didi-Huberman, Georges (2007): Bilder trotz allem. München: Wilhelm Fink.

Garcia, Luis Ignacio / Longoni, Ana (2012): Imágenes invisibles. Acerca de las fotos de desaparecidos. In: grumo 9, 12-21.

Gorini, Ulises (2008): La otra lucha. Historia de las Madres de la Plaza de Mayo (19831986). Buenos Aires: Grupo Editorial Norma.

Huffschmid, Anne (Hg.) (2006): Stadt als Labor: Krise und Erinnerung in Berlin und Buenos Aires. Berlin: Parthas.

Huffschmid, Anne / Durán, Valeria (Hg.) (2012): Topografías Conflictivas. Memorias, espacios y ciudades en disputa. Buenos Aires: Nueva Trilce.

Kracauer, Siegfried (2005 [1964]): Theorie des Filmes. Die Errettung der äußeren Wirklichkeit. Suhrkamp: Frankfurt am Main.

Silva, Armando (2006 [1992]): Imaginarios Urbanos. Bogota: Editorial Nomos.

Silva, Armando (Hg.) (2003): Urban imaginaries from Latin America. Ostfildern-Ruit: Hatje Cantz. 